\title{
Bilateral Optic Disc Edema Associated with Pyogenic Spondylitis
}

\author{
장지혜 ${ }^{1}$, 강승일 ${ }^{2}$, 김유철 ${ }^{1}$ \\ Ji Hye Jang ${ }^{1}$, Sung II Kang ${ }^{2}$, Yu Cheol Kim \\ 1계명대학교 의과대학 안과학교실, ${ }^{2}$ 제일안과병원 \\ 'Department of Ophthalmology, Keimyung University School of Medicine, Daegu, Korea \\ ${ }^{2}$ Cheil Eye Hospital, Daegu, Korea
}

Purpose: To report an uncommon case of bilateral optic disc edema as a complication of cervical pyogenic spondylitis.

Case summary: A 48-year-old male patient was diagnosed with C2 spondylodiscitis with direct extension from a retropharyngeal abscess. He had received intravenous nafcillin for coverage against methicillin-sensitive Staphylococcus aureus. After 3 weeks, he complained of blurred vision in both eyes. Fundus examination showed bilateral optic disc swelling with peripapillary flame-shaped hemorrhages, radiating hard exudates at the papillomacular area. After 8 weeks of continuous antibiotic therapy, the patient recovered slowly.

Conclusions: Involvement of the bilateral optic nerve with the cervical pyogenic spondylitis is uncommon. If a patient with the cervical pyogenic spondylitis complains of blurred vision or other ocular symptoms, ophthalmologists and neurologists should be aware of ocular complications.

Keywords: Optic disc; Retropharyngeal abscess; Spondylitis

\section{Introduction}

Pyogenic spondylitis is a rare but important spinal infection of the vertebral body involving the processes of the spine and intervertebral disc, which extend into the epidural and paraspinal spaces. Pyogenic spondylitis arises from a hematogenous spread of bacteria, most commonly of Staphylococcus aureus and Streptococcus species [1]. Incidence of this condition has been on the rise, possibly due to the improved life expectancy of patients with chronic diseases, malignancies and immune-deficiency diseases [2]. Involvement of the cervical spine (C-spine) in pyogenic spondylitis is uncommon and exhibits poor clinical outcomes such as abscess formation, spread of infection, permanent neurologic deficits, neck instability and torticollis [3]. To the best of our knowledge, there is no case report of optic nerve involvement associated with pyogenic spondylitis. However, we encountered a case of bilateral optic disc edema as a complication of $\mathrm{C}$-spine pyogenic spondylitis, which is described herein with a review of the relevant literature.

\section{Address reprint requests to Yu Cheol Kim, MD, PhD}

Department of Ophthalmology, Keimyung University Dongsan Medical Center, \#1035 Dalgubeol-daero, Dalseogu, Daegu 42601, Korea

Tel: 82-53-258-7855

E-mail: eyedr@dsmc.or.kr
Received: 2020. 2. 28

Revised: 2020. 3. 18

Accepted: 2020. 3. 26 


\section{Case Report}

A 48-year-old male patient with history of diabetes mellitus was referred to our ophthalmology department from the neurosurgical department. The patient was diagnosed with $\mathrm{C} 2$ spondylodiscitis due to a direct extension from a retropharyngeal abscess based on head and neck magnetic resonance imaging (MRI) (Fig. 1). He was hospitalized for 2 months with absolute bed rest and received intravenous

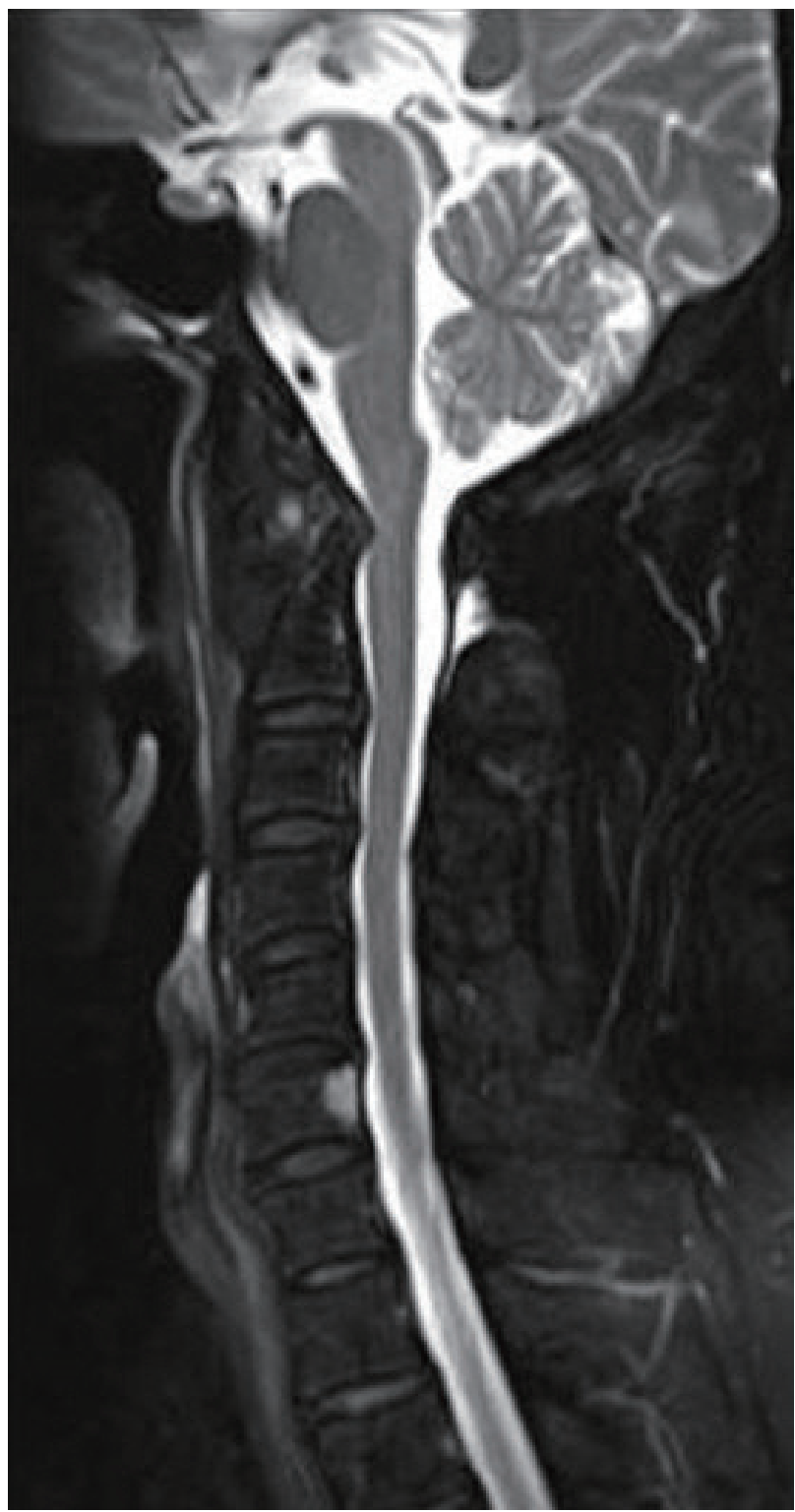

Figure 1. T2-magnetic resonance imaging image shows C2 spondylodiscitis due to direct extension from a retropharyngeal abscess. nafcillin 2 g per 4 hours for coverage against methicillin-sensitive Staphylococcus aureus. After 3 weeks of treatment, he started to exhibit blurred vision and metamorphopsia of the left eye. His best-corrected visual acuity was 20/20 in the right eye and 20/32 in the left eye. Anterior segment and vitreous showed normal findings. Relative afferent pupillary defect was not identified. Fundus examination revealed bilateral disc swelling with peripapillary flame-shaped hemorrhages. Additionally, radiating hard exudates were found at papillomacular area (Fig. 2). Optical coherence tomography also revealed optic disc swelling with hyper-reflective hard exudates (Fig. 3A). Fluorescein angiography (FAG) showed hyperfluorescence at the optic disc with peripapillary hypofluorescence (Fig. 3B). There was no finding of microaneurysm or similar defects on FAG. Indocyanine green angiography presented hypofluorescence at the optic disc margin (Fig. 3C). He had a highly elevated Erythrocyte sedimentation rate (ESR) level $(71 \mathrm{~mm} / \mathrm{h}$, normal ESR $<20$ $\mathrm{mm} / \mathrm{h}$, highly elevated ESR $\geq 60 \mathrm{~mm} / \mathrm{h}$ ), while his C-reactive protein (CRP) level was within normal range $(0.98 \mathrm{mg} /$ $\mathrm{L}$, normal CRP $<10 \mathrm{mg} / \mathrm{L}$, highly elevated $\mathrm{CRP} \geq 60 \mathrm{mg} / \mathrm{L}$ ). Follow up head and neck MRI remained unchanged.

The patient has fully recovered following the eight weeks of antibiotic regimen. After two months, his best-corrected visual acuity had improved to $20 / 20$ in both eyes. The bilateral optic disc swelling took more than two months to completely resolve. Fundus photography showed decreased edema and hemorrhage around the optic disc and absorbed hard exudates in the fovea (Fig. 4A). Humphrey perimetry test showed normal results in the right eye and a slight enlargement of blind spots in the left eye (Fig. 4B). Follow- up measurements of ESR and CRP levels were normal.

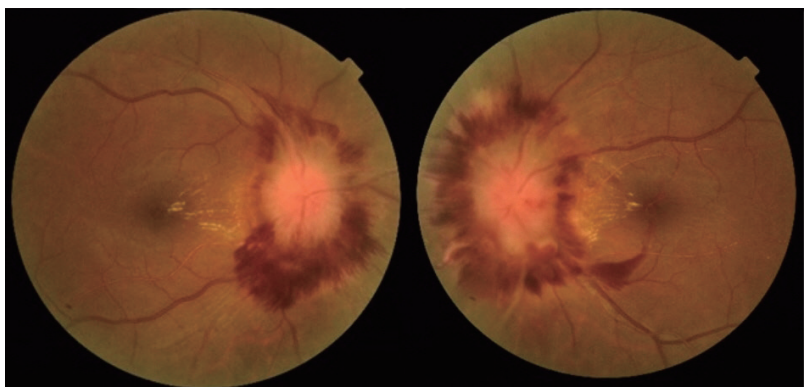

Figure 2. Fundus photograph of both eyes shows disc swelling with flame-shaped retinal hemorrhage and radiating hard exudates at papillomacular area. 

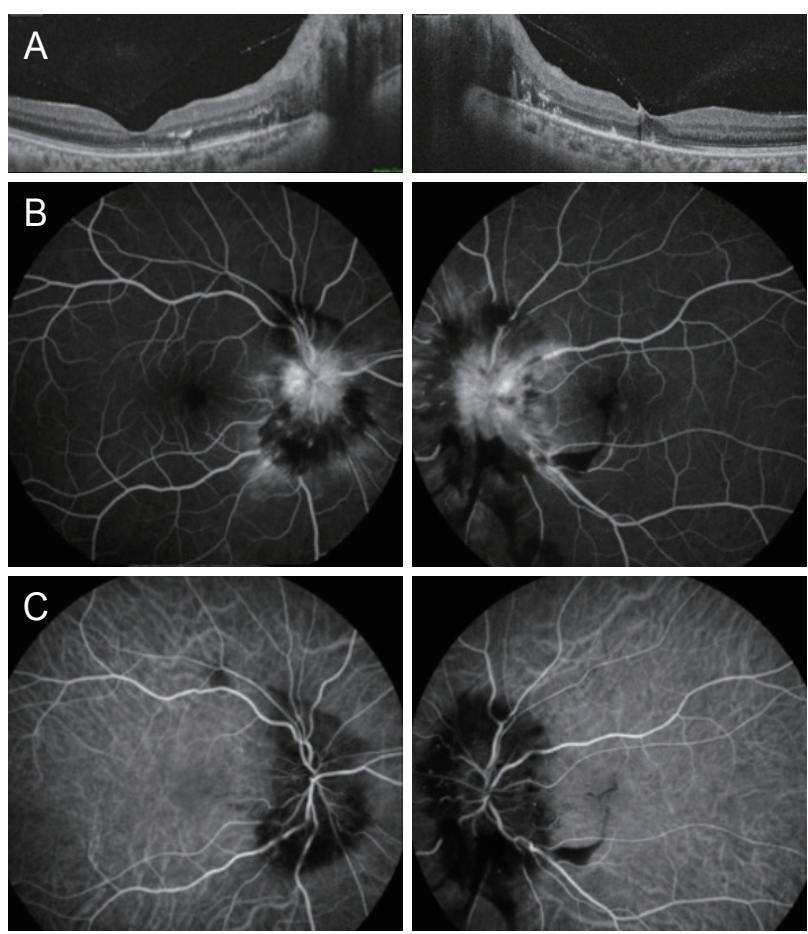

Figure 3. (A) Optical coherence tomography finding of the both eyes showing extensive optic edema with hyperreflective signals on the outer retinal layer. (B) Fluorescein angiographic images of both eyes show hyperfluorescence in the optic disc with parapapillary hypofluorescence. (C) Indocyanine green angiographic images show hypofluorescence in the optic disc margin of both eyes.

\section{Discussion}

Our patient had bilateral optic disc swelling and peripapillary flame-shaped hemorrhages related to cervical pyogenic spondylitis and retropharyngeal abscess. After intravenous antibiotic regimen treatment, bilateral optic disc swelling was resolved and visual acuity was recovered.

Cervical pyogenic spondylitis is uncommon compared to other types of vertebral pyogenic spondylitis [3], and accounts for $3-5 \%$ of all cases of osteomyelitis [4]. The source of cervical spine infection may often be obscure. According to Romagna et al. [5], 29\% of patients had a bacterial oral cavity infection, which appears to be a frequent source of pyogenic spondylitis. Likewise, our case had a methicillin-sensitive Staphylococcus aureus infection and C2 spondylodiscitis due to direct extension from a retropharyngeal abscess.

Several previous studies have reported of severe complica-
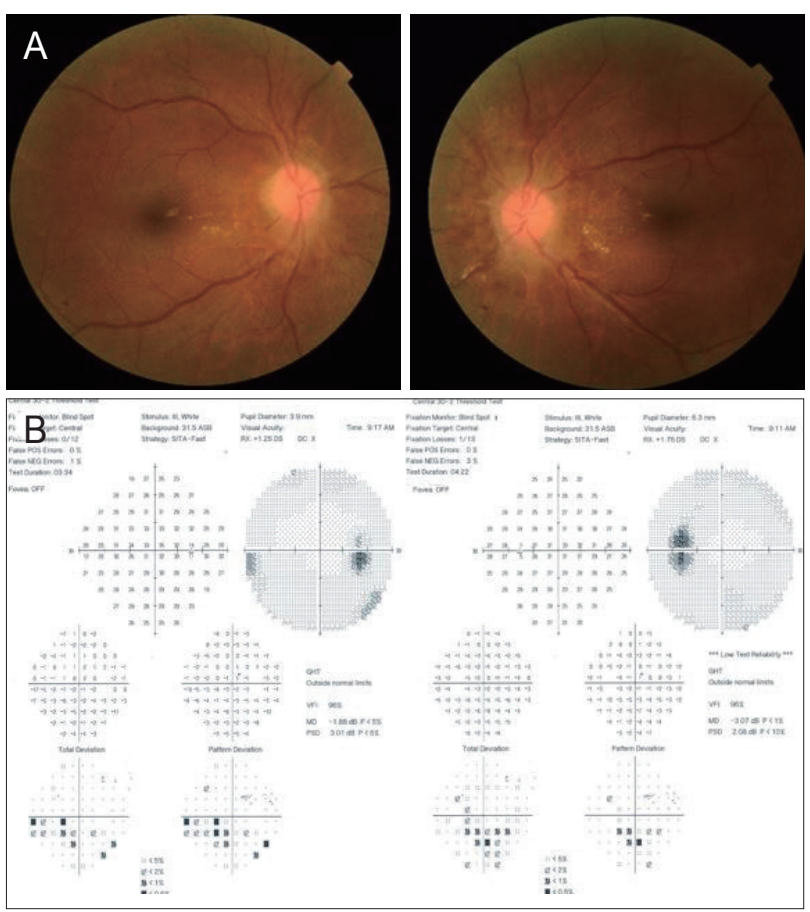

Figure 4. (A) Fundus photography of both eyes two months later shows that disc hemorrhages and swelling are mostly absorbed. (B) Humphrey perimetry shows normal results in the right eye and a slight enlargement of blind spots in the left eye.

tions due to pyogenic spondylitis of the cervical spine, such as epidural abscess, intraspinal abscess, atlantoaxial subluxation, and torticollis $[3,6-8]$. To the best of our knowledge, no cases of optic disc swelling due to pyogenic spondylitis of the cervical spine have previously been reported.

How can bilateral optic neuropathy occur in cervical pyogenic spondylitis? There are two possible mechanisms involved in disc swelling. The first is chronic increase of intra-cranial pressure. According to Hayreh [9], papilledema occurs as a result of chronic intracranial hypertension as opposed to acute rise in intra-cranial pressure. This is just a mechanical phenomenon. A slow rise in cerebrospinal fluid pressure in the optic nerve sheath, which produces axoplasmic flow stasis, results in swelling of the optic disc. Swelling of the optic disc consequently compresses the small vessels of the optic disc region causing parapapillary hemorrhage and venous engorgement. Riva et al. [10] also reported that optic disc swelling causes a reduction in the auto-regulatory capacity of the optic disc due to venous insufficiency.

The second mechanism of optic disc swelling is a direct spread of infection or indirect effect by inflammation. 
Cheung and Luk [1] reported that pyogenic spondylitis commonly arises from hematogenous spread of bacteria. Especially in the case of cervical spine infection, the pre-vertebral pharyngeal venous plexus may act as a route for the spread of bacteria.

ESR and CRP acts as non-specific inflammatory markers. ESR is normalized within several weeks while CRP levels stabilize within 7 days after resolution of tissue injury [11]. In our case, the patients had elevated ESR value. GroenHakan et al. [12] reported that patients with infectious uveitis showed normal ESR and CRP levels, patients with ocular inflammation occurring concurrently with bacteremia had highly elevated ESR and /or CRP.

In our case, we did not have information about the intracranial pressure level. However, we guess that severe bilateral disc edema and complete recovery of visual function after antibiotics treatment imply that the disc swelling may have been bilateral optic nerve inflammation with bacteremia rather than direct invasion. Additionally, the proximity of the abscess to the optic nerve does not seem to be the causative factor for the direct invasion, as bilateral involvement of the optic nerve and disc is rare.

In conclusion, the authors report a case of papilledema caused by C-spine pyogenic spondylitis, which is extremely rare. Ophthalmologists and neurologists should be aware of this complication of $\mathrm{C}$-spine pyogenic spondylitis, as close follow-up until recovery is required.

\section{Conflicts of Interest}

The authors declare that there is no conflict of interest.

\section{References}

1. Cheung WY, Luk KDK. Pyogenic spondylitis. Int Orthop 2012;36:397-404

2. Kehrer M, Pederson $C$, Jensen TG, Lassen AT. Increasing incidence of pyogenic spondylodiscitis: a 14-year population-based study. J Infect 2014;68:313-20.

3. Urrutia J, Zamora T, Campos M. Cervical pyogenic spinal infections: are they more severe diseases than infections in other vertebral locations? Eur Spine J 2013;22:2815-20.

4. Fantoni M, Trecarichi EM, Rossi B, et al. Epidemiological and clinical features of pyogenic spondylodiscitis. Eur Rev Med Pharmacol Sci 2012;16 Suppl 2:2-7.

5. Romagna A, Troeltzch M, Birkenmaier C, et al. Oral cavity infection: an underestimated source of pyogenic spondylodiscitis. J Neurol Surg A Cent Eur Neurosurg 2018;79:218-23.

6. Miyazaki M, Yoshiiwa T, Kodera R, Tsumura H. Clinical features of cervical pyogenic spondylitis and intraspinal abscess. J Spinal Disord Tech 2011;24:E57-61.

7. Tsunoda $K$, lizuka $H$, Sorimachi $Y$, et al. Atlanto-axial subluxation after pyogenic spondylitis of the atlanto-occipital joint. Eur Spine J 2011;20:S253-7.

8. Herman MJ. Torticollis in infants and children: common and unusual causes. Instr Course Lect 2006;55:647-53.

9. Hayreh SS. Pathogenesis of optic disc edema in raised intracranial pressure. Prog Retin Eye Res 2016;50:108-44.

10. Riva CE, Hero M, Titze P, Petrig B. Autoregulation of human optic nerve head blood flow in response to acute changes in ocular perfusion pressure. Graefes Arch Clin Exp Ophthalmol 1997;235:618-26

11. Jain S, Gautam V, Naseem S. Acute-phase proteins: as diagnostic tool. J Pharm Bioallied Sci 2011;3:118-27.

12. Groen-Hakan F, Eurelings L, van Laar J, Rothova A. Relevance of erythrocyte sedimatation rate and C-reactive protein in patients with active uveitis. Graefes Arch Clin Exp Ophthalmol 2019;257:175-80 